\title{
Frank Schlöffel
}

\section{Zionismus und Bibliophilie}

\section{Heinrich Loewe und die neuen ,Soncinaten`}

Am 15. Mai 1924 konstituierte sich in Berlin unter großer Beachtung der Presse ${ }^{2}$ die erste bibliophile jüdische Gesellschaft der Geschichte - die Soncino-Gesellschaft der Freunde des jüdischen Buches. Auch nach 1933 blieb die Vereinigung im Gegensatz zu anderen signifikanten Zusammenschlüssen, die sich im Jahr der Machtübernahme der Nationalsozialisten auflösten beziehungsweise aufgelöst wurden, ${ }^{3}$ noch einige Zeit tätig. Wie Josef Altmann vermutete, sollte sie unter Aufsicht der Gesellschaft der Bibliophilen in Weimar gestellt werden. ${ }^{4}$ Wenn der Schriftführer der Soncino-Gesellschaft, Herrmann Meyer, in einem Erinnerungsfragment schreibt, dass Soncino 1933 als „staatsfeindlich aufgelöst wurde“5, spricht daraus weniger historische Realität als vielmehr die Unzufriedenheit mit der Entwicklung der Gesellschaft nach 1933. Zahlreiche zentrale Akteure der Soncino-Gesellschaft flüchteten und verschoben damit ihre Lebensmittelpunkte sowie Aktionsräume. Heinrich Loewe, ihr langjähriger Vorsitzender (1926-1933), plante schon 1935 die Neugründung einer Gesellschaft der Freunde des jüdischen Buches in Tel Aviv. ${ }^{6}$ Auch machte sich augenscheinlich Enttäuschung über die neue Leitung der Gesellschaft bemerkbar, die ab 1933 Walther Michaelis oblag. Obwohl dieser Mitglied in der Maximilian-Gesellschaft, Vorstandsmitglied des Berliner Bibliophilen-Abends und des Fontane-Abends war, schrieb Josef Altmann im Januar 1938 an Loewe: „Es war ein grosser Fehler[,] diesen völlig an

1 Bei dem vorliegenden Artikel handelt es sich um eine überarbeitete Fassung des Kapitels „Die neuen ,Soncinaten““ aus meiner laufenden Dissertation, „Heinrich Eljaqim Loewe (1869-1951). Netzwerke und Räume“ an der Philosophischen Fakultät der Universität Potsdam. Das Buch wird voraussichtlich im Herbst 2015 im Neofelis Verlag erscheinen.

2 Vgl. im Anhang: Die Soncino-Gesellschaft in der Presse ihrer Zeit.

3 Siehe hierzu Fischer, Ernst: Zerstörung einer Buchkultur. Die Emigration jüdischer Büchersammler aus Deutschland nach 1933 und ihre Folgen: www.bibliophilie.de/index2.html?fischer. html main (03.12.2012).

4 Vgl. Brief von Josef Altmann an Heinrich Loewe vom 15.02.1934. Beit Ariela (BA), Boxnr. 7; Brief von Heinrich Loewe an Josef Altmann vom 06.03.1934. Zionistisches Zentralarchiv (CZA), A146/122. Zur Umstrukturierung der bibliophilen Gesellschaften nach 1933, vgl. Hansert, Andreas: Georg Hartmann (1870-1954). Biografie eines Frankfurter Schriftgießers, Bibliophilen und Kunstmäzens. Wien/Köln/Weimar 2009, S. 115-116.

5 Zitiert nach Fischer, Zerstörung einer Buchkultur.

6 Vgl. Brief von Heinrich Loewe an Kurt Freyer vom 11.03.1935. BA, Boxnr. 7; Brief von Heinrich Loewe an Alexander Marx vom 09.04.1935. BA, Boxnr. 7. 
hebr[äischen] $u[n d]$ jüd[ischen] Dingen uninteressierten Leuten die Gesellschaft zu überlassen."

Der Bücher- und Kunstsammler Herrmann Meyer, der bis zu seiner Auswanderung über Amsterdam nach Palästina im Jahr 1934 Geschäftsführer blieb, war zweifelsohne ihr Motor, er war Heinrich Loewe zufolge ihre „Seele“8. In seinen Erinnerungen berichtet Loewe, wie Meyer zu ihm kam und die Gründung einer jüdischen bibliophilen Gesellschaft vorschlug. Er hätte sich gern bereit erklärt, Meyer nicht nur auf der Suche nach potentiellen Interessenten in zionistischen, Bundesbrüder- ${ }^{9}$ und sonstigen Kreisen behilflich zu sein, sondern sagte auch seine persönliche Mitarbeit zu. Tatsächlich gehörten später bekannte Zionisten zu den Mitgliedern der Gesellschaft: die Philosophen Martin Buber und Franz Rosenzweig, der Publizist Felix Theilhaber, der Rechtsanwalt und beliebte Volksredner Alfred Klee, der Redakteur und Verleger Siegmund Kaznelson, der Buchhändler Ludwig Mayer, der Historiker und Archivar Georg Herlitz, der Schriftsteller Sammy Gronemann und auch Chaim Weizmann, der Präsident der Zionistischen Weltorganisation. Zu den in Palästina lebenden Mitgliedern gehörten der Dichter Chaim Nachman Bialik, der Gründer des Jewish Colonial Trust Isaac Goldberg, der Direktor der Anglo-Palestine Bank Siegfried Hoofien sowie der Arzt und Moses Hess-Biograph Theodor Zlocisti.

Heinrich Loewe nimmt in diesem Kreis eine herausragende Rolle ein. 1869 im Ackerbaustädtchen Wanzleben bei Magdeburg geboren, siedelte er 1889 nach Berlin über. ${ }^{10}$ Ursprünglich hatte er seine Reise angetreten, um zu studieren - Loewe immatrikulierte sich an der Philosophischen Fakultät der Friedrich-Wilhelms-Universität und besuchte Veranstaltungen an der Hochschule für die Wissenschaft des Judentums. Gleichzeitig knüpfte er Kontakte zu in Berlin aktiven zionistischen, vornehmlich aus Osteuropa stammenden Studenten. Er gehörte als einziger deutscher Jude zu dem 1889 gegründeten Russisch-jüdischen wissenschaftlichen Verein, dem ersten zionistischen Zirkel im Deutschen Reich, und war 1892 einer der Gründer des Vereins Jung Israel. In der folgenden Dekade war er als Schriftleiter an der inhaltlichen Gestaltung diverser zionistischer Periodica beteiligt, unter anderem als Chefredakteur der Jüdischen Rundschau (1902-1908), dem Zentralorgan der Zionistischen Vereinigung für Deutschland, die sich unter dem Namen National-Jüdische Vereinigung Köln bereits im Vorfeld des 1. Zionistenkongresses in Basel von 1897 als einer von zahlreichen zionistischen Landesverbänden gegründet hatte.

7 Brief von Josef Altmann an Heinrich Loewe vom 12.01.1938. BA, offener Bestand.

8 Loewe, Heinrich: Sichronot Kap. Die Soncino Gesellschaft. CZA, A146/75, S. 1.

9 Vgl. ebenda.

10 Siehe hierzu Schlöffel, Frank: „Heinrich Eljaqim Loewe (1869-1951). Netzwerke und Räume“, Kap. 3 „Nationaljudentum in Aktion“. 
Trotz intensiver Bemühungen Loewes ab Frühjahr 1898 gelang es ihm nicht, eine bezahlte Anstellung in einer der im Entstehen begriffenen zionistischen Institutionen zu finden. ${ }^{11}$ Er war deshalb gezwungen, seine wirtschaftliche Existenz außerhalb jener Unter- und An-Organisationen der Zionistischen Weltorganisation zu sichern. Schließlich bewarb sich Loewe erfolgreich auf eine Stelle an der Königlichen Universitätsbibliothek zu Berlin, wo er, im September 1905 als Beamter vereidigt, bis zu seiner Übersiedlung nach Tel Aviv im Jahr 1933 - zuletzt als Bibliotheksrat - tätig war. Anlässlich des 7. Zionistenkongresses, der drei Monate nach Loewes Vereidigung stattfand, veröffentlichte er sogleich eine Broschüre unter dem Titel Eine jüdische Nationalbibliothek ${ }^{12}$, die deutlich machte, wo sich Loewe innerhalb des zionistischen Diskurses zukünftig verorten werde. Die jüdische Nationalbibliothek, die 1930 als Jüdische National- und Universitätsbibliothek (JNUL) auf dem Skopusberg in Jerusalem eröffnet und unter wesentlicher Beteiligung Heinrich Loewes konzeptioniert und gebaut wurde, ${ }^{13}$ gehörte ebenso zu den zentralen Betätigungsfeldern Loewes wie eine dezidiert hebräische Kulturarbeit, die er jahrzehntelang bemüht war, in die Agenda des zionistischen Kollektivs einzuschreiben. Welche Rolle Loewe in Bezug auf die hebräische Kulturarbeit vor allem in der jüdischen Diaspora spielte, lässt eine 1913 in der Welt zitierte Mitteilung aus der hebräischsprachigen Zeitschrift Ha-Zeman erahnen:

Der deutsche Zionismus hat schon eine mehr als fünfzehnjährige Vergangenheit hinter sich, und noch immer gibt es unter den deutschen Zionisten keine Leute, die hebräisch lesen und verstehen, die sich für unsere Literatur interessieren und sie fördern. Nur auf einen einzigen unter ihnen kann man hinweisen, der die hebräische Sprache erlernt hat, der aber darin keine Nachfolge gefunden hat: Dr. Heinrich Loewe. ${ }^{14}$

Dem Buch indes als kleinster Einheit innerhalb des von Loewe propagierten, auf die „kulturelle Hebung“ Palästinas abzielenden jüdischen Bibliothekswesens ${ }^{15}$ weist er im Rahmen seiner Bibliotheksphilosophie schon 1902 eine spezifische Rolle zu. ${ }^{16}$ Er stellte es als „Ehrenpflichtexemplar“ zur Schau, als Brücke zwischen Bücherspendern und Bibliothek. Jedes gespendete Buch solle ein Exlibris

11 Ebenda, Kap. 4.1 „Die Neuvermessung des zionistischen Terrains Berlin nach dem 1. Zionistenkongress".

12 Loewe, Heinrich: Eine Jüdische Nationalbibliothek. Berlin 1905.

13 Siehe Schlöffel, Heinrich Eljaqim Loewe, Kap. 7.5 ,Jüdische National- und Universitätsbibliothek“. 14 N.N.: Revue der Presse. In: Die Welt (28.02.1913), S. 277.

15 Siehe Kirchhoff, Markus: Häuser des Buches. Bilder jüdischer Bibliotheken. Leipzig 2002, S. 65-71 [Kap. ,Jüdisches Bibliothekswesen im Lande Israel (Heinrich Loewe)“].

16 Siehe hierzu Loewe, Heinrich: Bemerkungen über die Nationalbibliothek in Jerusalem. In: Jüdische Rundschau 42 (15.10.1902), S. 19-21, hier S. 21. 
als sichtbares Zeichen enthalten, „das den Namen des Spenders für alle Zeiten mit dem Buche vereinigt und ihm ein ewiges Andenken in Jerusalem sichert“17.

Diese Praxis, Bücher mit Exlibris zu versehen, legte er nicht nur im Zuge der Sammlung für die JNUL an den Tag, die er nach 1914 als Leiter der von der zionistischen Exekutive in Berlin gegründeten Hauptsammelstelle dirigierte. Sie fand offenbar ebenso Eingang in seine private Büchersammeltätigkeit. Verfügte jedoch ein Teil der ersten Mitglieder der Soncino-Gesellschaft über mit bibliophilen Kostbarkeiten gespickte Bibliotheken, kann Loewe keineswegs als ein im klassischen Sinne Bibliophiler bezeichnet werden. Loewes Privatbibliothek, die „Bibliothek eines armen Mannes“18, wie er sie selbst beschrieb, war mehr Arbeitsbibliothek, eine Gebrauchsbibliothek zur jüdischen, vor allen Dingen zur zionistischen Geschichte und Kultur, die nur in seltenen Fällen - etwa im Fall der SoncinoVeröffentlichungen, die Loewe freilich in die Sammlung aufnahm - bibliophile Züge aufwies. Am Inhalt der meisten Bücher, welche zuletzt die Regale seiner Wohnung in der Flemingstraße füllten, hatte er ein konkretes Erkenntnisinteresse. Büchersammeln diente ihm als Erwerb von Wissen und seiner Klassifizierung. Als „Kampf gegen die Zerstreuung“"19 lässt sich mit Walter Benjamin die Sammelpraxis Loewes lesen, die schon 1914 im Rahmen der Propaganda für die JNUL stark gemacht wurde: „Können wir auch nicht alle Zerstreuten Israels im Lande der Väter sammeln, aber die zerstreuten Bücher Israels, die überall herumgetragenen Gedanken des hebräischen Geistes zu sammeln, dazu sind wir imstande“, heißt es hier. ${ }^{20}$ Auch mit seiner Privatbibliothek schaffte Loewe einen Ort, der das Periphere in einem Zentrum vereint, der die jüdische Welt von gestern versammelt, um die Welt von heute zu deuten und sich gegen die Welt von morgen abzusichern. ${ }^{21}$

17 Loewe, Heinrich: Eine hebräische Universitätsbibliothek zu Jerusalem. In: Jüdische Rundschau 23 (05.06.1914), S. 242-243, hier S. 243.

18 Loewe, Heinrich: Sichronot. Kap. Aus meiner Bibliothek. CZA, A146/168, S. 1.

19 Benjamin, Walter: Das Passagen-Werk. Gesammelte Schriften, Bd. V.1, hrsg. v. Rolf Tiedemann. Frankfurt am Main 1982, S. 279.

20 N.N.: Jüdische Kulturarbeit. Der Jüdische Kulturfonds Kedem, "קרן התרבות הדעברית "קדם. Berlin 5674 [1913], S. 14. Zum Motiv der Zerstreuung, ferner zum Verhältnis von Sammeln, Totalität und Fragment siehe Weinberg, Manfred: Ein Seismograph für geistigen Erbgutsverkehr. Sieben Bemerkungen zur Kulturwissenschaftlichen Bibliothek Aby Warburgs sowie zu Sammeln, Bibliophilie und Exzentrik. In: Sammler, Bibliophile, Exzentriker, hrsg. von Aleida Assmann, Monika Gomille u. Gabriele Rippl. Tübingen 1998, S. 117-138.

21 Siehe hierzu Stagl, Justin: Homo Collector. Zur Anthropologie und Soziologie des Sammelns. In: Sammler, Bibliophile, Exzentriker, S. 37-54, hier S. 39-40. 


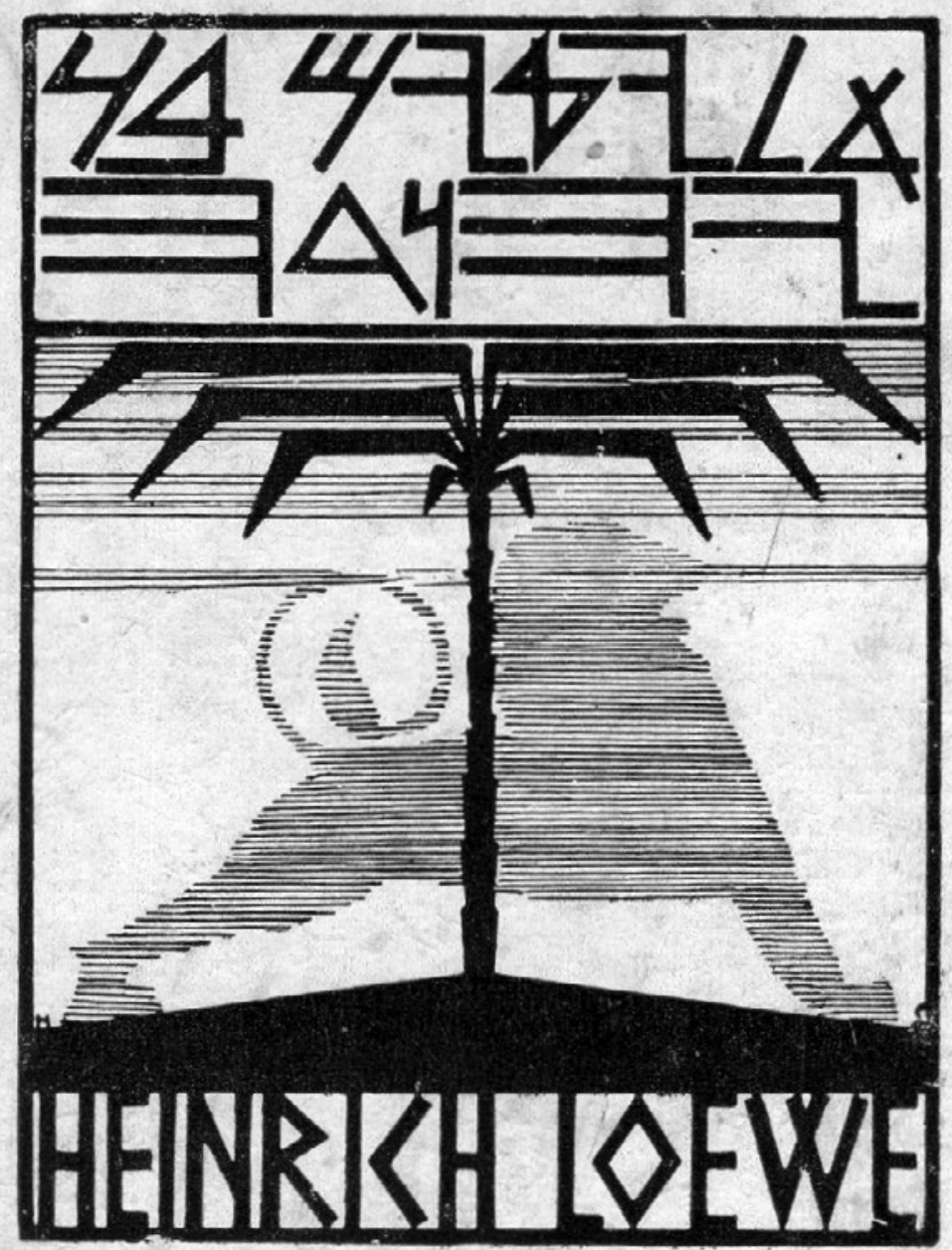

Abb. 8: Exlibris von Heinrich Loewe.

Die Satzung der Soncino-Gesellschaft, die von den zukünftigen Vorständen verabschiedet wurde und zeigt, dass die Gesellschaft sich offensichtlich zunächst, die zionistische Kulturagenda stärker affirmierend, „Gesellschaft der Freunde 
des hebräischen Buches“ nennen wollte, ${ }^{22}$ trat knapp einen Monat nach der konstituierenden Versammlung am 17. Juni 1924 durch Eintragung des Vereins beim hiesigen Vereinsregister in Kraft. Das Hauptziel der Soncino-Gesellschaft bestand in der Schaffung einer “neu-alten“ Form des jüdischen Buches, der „Herausgabe seltener Texte und wertvoller Drucke von Werken jüdischen Inhalts und jüdischen Geistes unter besonderer Berücksichtigung von hebräischen Werken“ wolle man sich widmen, heißt es in den Vereinsstatuten. ${ }^{23}$ In einem Zirkular, das 1924 an Mitglieder in zionistischen Organisationen versandt wurde, präzisierte der Vorstand dahingehend und verwob die Idee der „kulturellen Hebung“, die Loewe bereits in seinen Entwürfen für die zeitgenössische jüdische Kulturgeographie Palästinas mit den Zielen des zionistischen Kollektivs verknüpfte, mit denen der Soncino-Gesellschaft. Auch die Vorstellung, Repräsentationsformen zu schaffen, die die „zivilisatorische Höhe“ adäquat abbilden würden, setzte auf jene tradierten zionistische Diskurselemente auf, die sich augenscheinlich mit jenen der deutschen Bibliophilenbewegung im Programm der Gesellschaft verwoben $^{24}$ :

Die Soncino-Gesellschaft, deren Bestand bereits durch eine Mitgliedschaft von über 200 Einzelpersonen und Bibliotheken gesichert ist, soll sich zu einem wichtigen Faktor innerhalb der allgemeinen jüdischen Kulturbestrebungen entwickeln; sie hat sich die Aufgabe gestellt, die jüdische Buchherstellung durch Beratung und Kritik auf den der jüdischen Allgemeinkultur entsprechenden Stand zu heben und dahin zu wirken, daß die Form des jüdischen Buches als repräsentativ für das jüdische Geistesleben gelten darf. ${ }^{25}$

22 Ein entsprechender Signet-Entwurf, der den Namen „Soncino-Gesellschaft der Freunde des hebräischen Buches“ führt, findet sich im Archiv des Jüdischen Museums Berlin (JMB), Sammlung Soncino-Gesellschaft, DOK 93/502/20-23.

23 Satzungen der Soncino-Gesellschaft der Freunde des jüdischen Buches vom 15.05.1924. Paragraph 2 Abs. b, JMB, Sammlung Soncino-Gesellschaft, DOK 93/502/1.

24 Siehe hierzu den Beitrag von Philipp Messner in diesem Band.

25 Werbebrief der Soncino-Gesellschaft der Freunde des jüdischen Buches, [1924]. JMB, Sammlung Soncino-Gesellschaft, DOK 93/502/7-14. Von diesem Zirkular wurden unterschiedliche Varianten angefertigt. Die endgültigen Textfassungen orientierten sich an den Adressaten des Schreibens. Im zitierten Brief, der - wie erwähnt - an zionistische Aktivisten geschickt wurde, verwies man darauf, dass man größtes Interesse an „kulturell interessierten Kreise[n] der zionistischen Bewegung“ hätte. Ferner bat man darum, in „Ihren Kreisen (Ortsgruppe, Zirkel, Verbindung) aktiv für einen Beitritt in die Gesellschaft zu werben“. Diese Textfassung wurde von Herrmann Meyer gezeichnet. Eine weitere von Siegfried Wolff und Moritz Simon gezeichnete Variante wurde an Mitglieder des Bnei Brit versandt (Werbebrief der Soncino-Gesellschaft der Freunde des jüdischen Buches, [1924]. JMB, Sammlung Soncino-Gesellschaft, DOK 93/502/15-19.) Ein weiteres, allgemein gehaltenes Anschreiben verwies als einziges darauf, dass die Gesellschaft „durch das Zusammenwirken kulturell interessierter Vertreter aller Richtungen des Judentums [Hervorhebung F.S.]“ entstanden sei. 
Hier wie in der Satzung wurde kodifiziert, was beispielsweise Chaim Nachman Bialik bereits 1913 implizit einforderte und als Zeitdiagnose der jüdischen Literatur festhielt:

Die jüdischen Literaturkenner wundern sich schon längst über eine sonderbare Erscheinung: unser Volk besitzt eine alte Literatur, die in sich die verschiedensten Literaturformen vereinigt und reichste Inhalte bietet; wir besitzen eine neue junge Literatur, die ebenfalls an hervorragenden Begabungen reich ist; wir haben auch viele wertvolle Werke von Juden in vielen fremden Sprachen - und trotz alledem vermögen wir nicht, einem gebildeten jüdischen Zeitgenossen wenigstens eine kleine Anzahl von Büchern in die Hand zu geben, die wert sind, von ihm liebgewonnen zu werden, so dass er sich in Stunden geistigen Hungers an ihnen sättigt. ${ }^{26}$

Besonders bemerkenswert ist der von Bialik aufgeworfene Topos der „Liebe zum Buch“. Dieser spielt auch in einem nach Gründung der Soncino-Gesellschaft entstandenen Manuskript Loewes eine zentrale Rolle. Als „,ihre Geliebte“ ${ }^{27}$ porträtiert er das Buch in Bezug auf die Gesellschaft, als Gegenstand, den man durch eine spezifische Formgebung die „Energie“ verleihe, positive Gefühle beim Lesen zu evozieren: „[M]itempfinden“ sollen „auch andere die Freude an der Schönheit“ 28 . Simhat Tora - die Freude der Tora, welche sich im gleichnamigen jüdischen Feiertag verkörpert - scheint hier als Archetypus jüdischer Buchliebe chiffriert zu sein, ein Bild, das Ludwig Blau, ebenfalls Mitglied der Soncino-Gesellschaft, deutlich herausarbeitete, indem er für die erste Ausgabe der Soncino-Blätter von 1925 mit Blick auf die Toraschreiber vergangener Jahrhunderte festhielt: „Die alten Freunde des hebräischen Buches schöpften gleichfalls ihre Buchliebe aus der Liebe zur Thora und ihrer sinnfälligen Erscheinung. “29

Dann baut Blau die Brücke in die Gegenwart, hin zur Soncino-Gesellschaft, dessen ehrgeizigstes Projekt in der ,mit den Mitteln unserer Zeit die uralte Wahrheit zum neuen Ausdruck“30 bringende Herstellung der Chamischa Chumsche Thorah, der Hebräischen Bibel, ${ }^{31}$ bestand:

Mögen nun die neuen ,Freunde des hebräischen Buches‘ zu Beginn ihrer Tätigkeit ihren Blick zu jenem Buche erheben, aus welchem letzten Endes jedes hebräische Buch hervorgesprossen

26 Bialik, Chaim Nachman: Das hebräische Buch. Gekürzte Uebersetzung aus dem Hebräischen (Monatsschrift Ha-Schiloach, Odessa Novemberheft 1913) von Baruch Krupnik. In: Neue Jüdische Monatshefte 4, 2/4 (1919), S. 25-37, hier S. 25.

27 Loewe, Heinrich: Das jüdische Buch. BA, Boxnr. 12, S. 4.

28 Ebenda.

29 Blau, Ludwig: Das Schreiben des Sefer Thora. In: Soncino-Blätter 1 (1925), S. 20.

30 Buber, Martin: [Notiz an die Soncino-Gesellschaft]. JMB, Sammlung Soncino-Gesellschaft, DOK 93/502/122.

31 Vergleiche hierzu den Beitrag von Philipp Messner in diesem Band. 


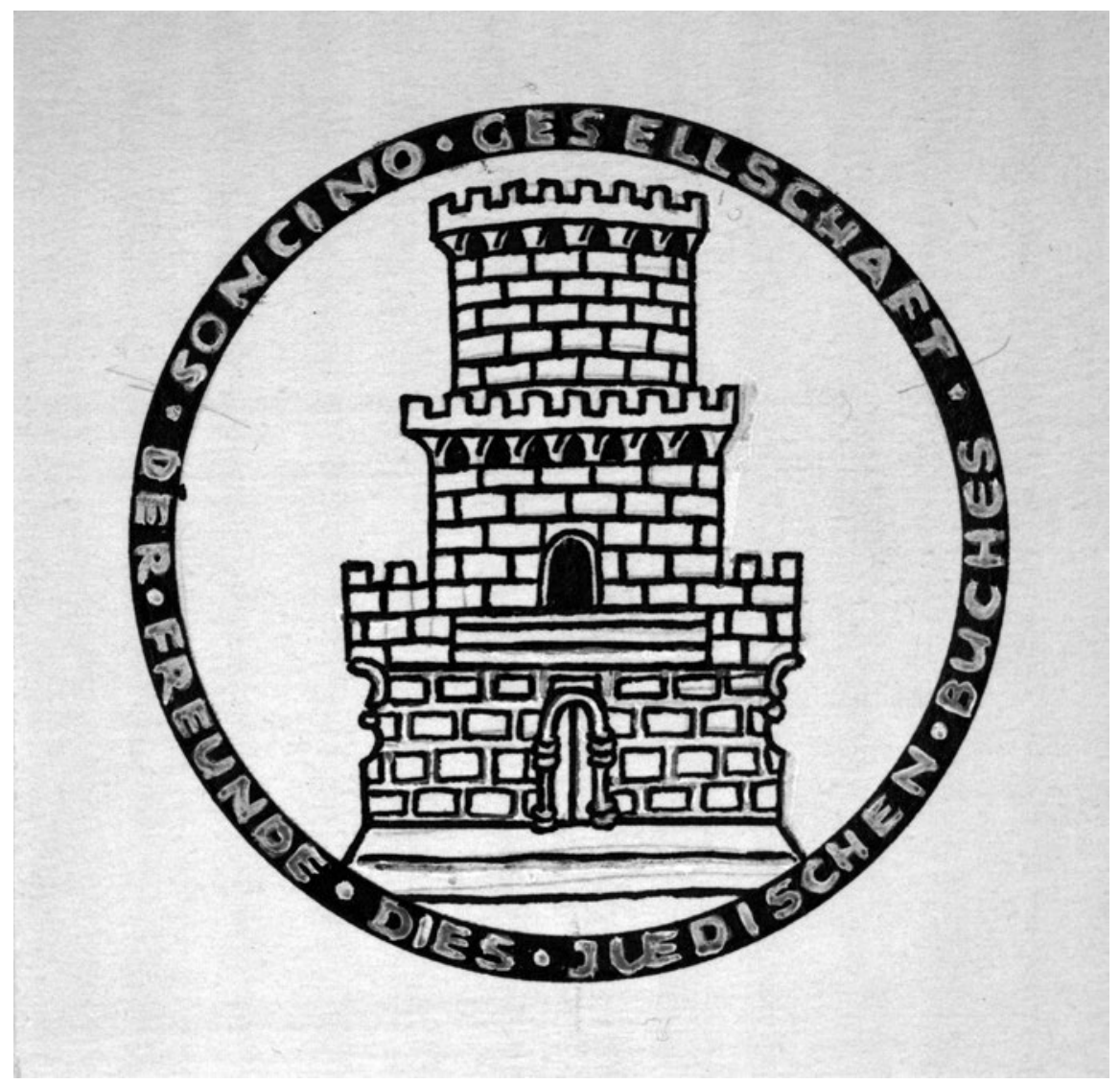

Abb. 9: Entwurf für das Signet der Soncino-Gesellschaft, um 1924.

ist, $\mathrm{zu}$ jener altehrwürdigen Buchrolle, welche den Jahrtausenden getrotzt und als einzige überlebende Vertreterin der alten Bücherwelt dasteht. Aus ihrem ewigen Bestande wollen wir Mut und Begeisterung, Ausdauer und Festigkeit zur vollen Erfüllung unserer idealen Aufgaben schöpfen, den alten Freundes des hebräischen Buches stets neue zu werben. ${ }^{32}$

Unter den Kennzeichen für eine adäquate Buchausstattung erlangte über den Einband und das Papier hinaus das Schriftbild - vor allem das hebräische Schriftbild - einzelner Publikationen der Soncino-Gesellschaft besondere Bedeutung. Während allerdings in den Straßen Palästinas, etwa an den Litfaßsäulen Tel Avivs, das Hebräische seine „mit der religiösen Tradition verbundene Gestalt

32 Blau, Das Schreiben des Sefer Thora, S. 20. 
hinter sich“33 ließ, war es erklärtes Anliegen einer Vielzahl von Buchprojekten der Gesellschaft, die Gestalt ausgezeichnet hergestellter, religiöser Druckwerke wiederzugeben, ihre Druckverfahren zu imitieren, sie zuweilen qualitativ zu übertreffen. Diese beabsichtigte Verknüpfung mit der Blütezeit der hebräischen Buchproduktion deutet sich an allererster Stelle im Namen der Gesellschaft an: Soncino. Die bekannte Druckerfamilie hatte sich Ende des 15. Jahrhunderts in dem oberitalienischen Städtchen Soncino niedergelassen und sich nach diesem benannt, dort eine ihrer vielen Druckereien errichtet und gehörte zu den ,großen Meister[n] aus der frühen Zeit der hebräischen Druckkunst“"34. Auch wählte man deren Signet - den Turm, der als charakteristisches Erkennungszeichen in simplifizierter Form sämtliche Drucke der Soncino-Gesellschaft zierte.

Den aktiven Kern der Gesellschaft bildete ihr Vorstand. Neben Herrmann Meyer und den beiden weiteren Initiatoren - den Verlegern Abraham Horodisch und Moses Marx - gehörten Josef Altmann, Willi David, Sammy Gronemann, Moritz Simon, Felix Struck, Siegfried Wolff und nicht zuletzt Heinrich Loewe zum ersten gewählten Vorstand. ${ }^{35}$ Dieser agierte Horodisch zufolge als „Arbeitsgremium“36, das heißt die Mehrzahl seiner Mitglieder war aktiv in Auswahl und Gestaltung der Soncino-Veröffentlichungen involviert. Auch Loewe, der nach dem plötzlichen Tod von Siegfried Wolff im März 1926 bis zu seiner Übersiedlung nach Tel Aviv den Vorsitz der Gesellschaft übernahm, war einer von denen, die nicht nur repräsentative Aufgaben übernahmen, etwa Einführungen und sonstige Redebeiträge auf den jährlichen Versammlungen leisteten, sondern der eigenverantwortlich Buchprojekte von der Idee bis zur Drucklegung betreute.

Die Soncino-Gesellschaft, die 1938 aufgelöst wurde, publizierte mehr als einhundert bibliophile Einblatt-, Gelegenheits- und Spendendrucke, Zeitschriftenbände,

33 Messner, Philipp: Tel Aviv und die Revolution des hebräischen Schriftbilds. In: Pardes 15 (2009), S. 22-38, hier S. 24.

34 Horodisch, Abraham: Ein Abenteuer im Geiste. Die Soncino Gesellschaft der Freunde des jüdische Buches. In: Bibliotheca Docet. Festgabe für Carl Wehmer, hrsg. von Siegfried Joost. Amsterdam 1963, S. 181-208, hier S. 182. Neben Soncino war Daniel Bomberg als Namensgeber im Gespräch. Der von Horodisch vorgeschlagene Bomberg war im Gegensatz zu den Soncinaten ein christlicher Drucker. Aus Antwerpen stammend, begann er Anfang des 16. Jahrhunderts mit der Drucklegung hebräischer Werke.

35 Bis zur offiziellen Gründung der Gesellschaft war Abraham Horodisch Vorsitzender. Ab spätestens 1928 waren anstelle von Willy David, Abraham Horodisch und Moses Marx, Menko Max Hirsch, Hofgerichtsrat Dr. Rosenberg und Max Scholem im Vorstand vertreten. Ein Jahr später kehrte Horodisch in den Vorstand zurück. Zudem wurden Martin Brunn, Gotthard Laske, Carl Lewin, Karl Schönberg, Max Strauß, Walther Michaelis und Erich Scholem in den Vorstand gewählt. Max Scholem und Rosenberg wurden damit ersetzt. Spätestens ab 1931 war auch Leo Heskel im Vorstand aktiv.

36 Horodisch, Abenteuer im Geiste, S. 183. 
Bücher und Vereinsdrucksachen. Besondere Bedeutung hatten die Sonderpublikationen, deren Ausgabe die Mitglieder Jahr für Jahr regelrecht zelebrierten: Die Ordentlichen Mitglieder der Gesellschaft trafen sich regelmäßig zu großen Versammlungen, die bereits ab 1926 jeweils an zwei Tagen im Jahr in Berlin stattfanden (ab 1931 tagte man auch anderenorts, etwa in Frankfurt am Main). Das Programm folgte dabei einem festgelegten Ablauf: Nach Ausgang des Shabbat begaben sich die Versammlungsteilnehmer zu einem „Begrüßungsabend“ in einen prachtvollen Berliner Saal. Erwähnt seien hier 1926 das Hotel Prinz Albrecht in der gleichnamigen Straße, 1929 die Städtische Oper (heute: Deutsche Oper) in der Bismarckstraße 34 und 1930 das Hotel Kaiserhof am Wilhelmplatz. Die Begrüßungsabende waren mit diversen Ansprachen und Vorträgen gefüllt. Am folgenden Tag fand die eigentliche Jahreshauptversammlung statt. Der Ansprache des Vorsitzenden folgten Verhandlungen von Vereinsinterna - etwa der Tätigkeitsbericht, Aussprachen und Mitteilungen. Den Höhepunkt der Jahreshauptversammlungen, die ab spätestens 1928 im Sitzungsaal der Repräsentantenversammlung der Berliner jüdischen Gemeinde stattfanden, bildete ein Festvortrag, der die Versammlung abschloss. Anschließend fanden sich die geladenen Gäste in einem anderen Saal zum „Gesellschaftsabend“ zusammen. Der Gesellschaftsabend, zu dem stets Abendgarderobe erbeten wurde, bestand aus einem Festessen und der Übergabe der an die Soncino-Gesellschaft gespendeten Sonderpublikationen. Gegen Vorlage der aufwendig gestalteten Ausweise erhielten die Gäste ihre Eintrittskarten. Diese mit Lochstreifen versehenen Ausweise umfassten unter anderen Coupons, gegen die die Teilnehmer nach dem Essen die bereitgestellten bibliophilen Drucke eintauschen konnten.

Mit insgesamt zehn Buchprojekten war Heinrich Loewe einer der herausragenden Autoren beziehungsweise Herausgeber der Soncino-Gesellschaft. Unter den bibliophilen Ausgaben, die Loewe abfasste oder redigierte, fanden sich einerseits volkskundliche Schriften, die sich entweder mit biblischen und religionsgeschichtlichen Themengebieten auseinandersetzen, sich der jüdischen Namensforschung, jüdischer Spielkultur oder dem jüdischen Humor widmeten. Ferner publizierte er vergleichsweise umfangreiche Würdigungen von Aaron Ember und Ignaz Goldziher. Weiterhin bearbeitete Loewe religiöse Texte, die als Reproduktionen veröffentlicht wurden, darunter eine bilinguale Ausgabe des „Goldenen Aleph-Beth“ und das wohl aufwendigste Buch Loewes für die Soncino-Gesellschaft, eine Faksimile-Ausgabe der Pessach-Haggadah des Gerschom Kohen mit kritisch kommentierendem Beiheft, ${ }^{37}$ die als erster Band der ehrgeizigen Buchreihe Monumenta Hebraica et Judaica publiziert wurde.

37 Die Pessach Haggadah des Gerschom Kohen, gedruckt zu Prag 5287 / 1527, hrsg. von Heinrich Loewe u. Benzion Katz. Berlin 1926. 


\section{A U S W E I S}

für

zur Teilnahme an dem aus Anlaß der 1. ordentl. Generalversammlung am Sonntag, den 24. Mai $19^{2} 5$ zu Berlin, im Hotel „Prinz Albrecht ${ }^{*}$ (A.Huster), Berlin SW 1 , Prinz-Albrecht-Straße 9, abends / $8 \mathrm{Uhr}$ stattfindenden

Fest-Essen und Gesellschafts-Abend

Es wird gebeten, diesen Ausweis sorgfiltig aufbewahren zu wollen, da ein Eratz für etwa abhanden gekommene Ausweise keinesfalls geleistet werden kann. - Gesellsehafts-Kleidung erbetes.

Marz \& Ca Berlin SW
Gegen Abgabe dieses Abschnittes wind in der Vorhalle des Hotels die Eintrittskarte ansgehindigt.

Nr198

Dieser Abschnitt ist nur Kontrolle der Teilnehmerahd bestimmt und wird wìhrend der Thel eingesammelt.

Gegen Abgabe des nachatehenden Coupons kōnnen die für die Mitglieder und Festteilnehmer gespendeten Sonderpublikationen in Emfang genommen werden. Während des Essens wird bekannt gegeben, welche Gaben fïr die einzelnen Abschnitte bestimmt sind.

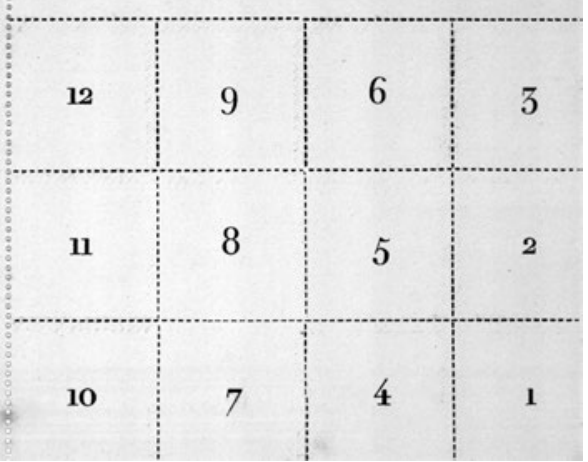

Abb. 10: Innenseite des Ausweises zur ersten ordentlichen Generalversammlung der SoncinoGesellschaft, 24. Mai 1925.

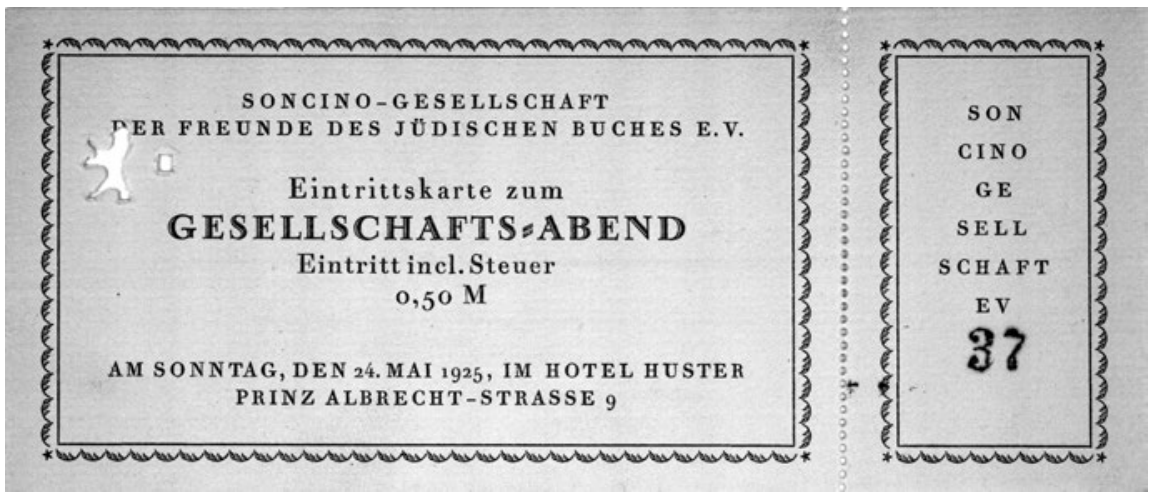

Abb. 11: Einlasskarte zum Gesellschafts-Abend der Soncino-Gesellschaft, 24. Mai 1925. 


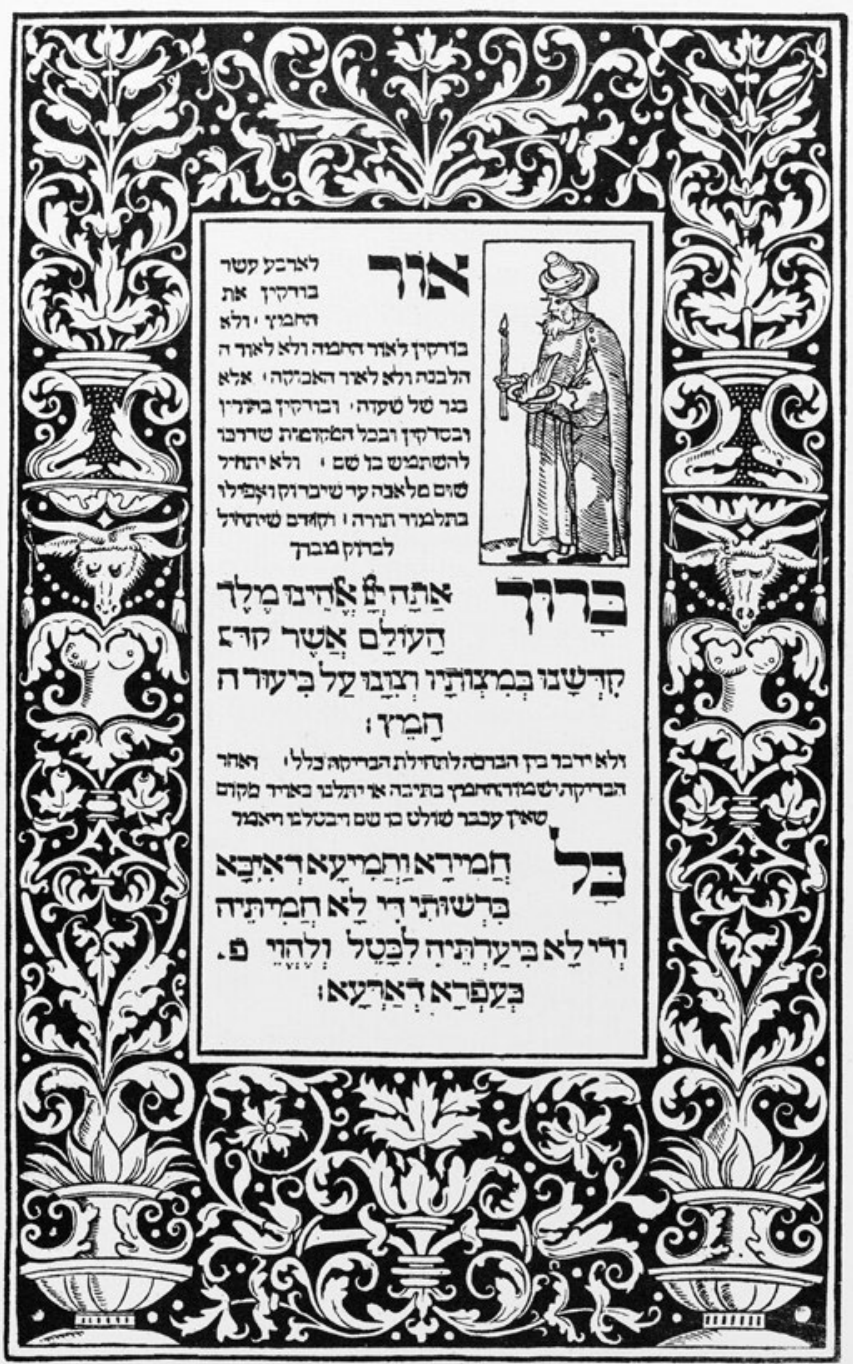

Abb. 12: Erste Seite der Faksimile-Ausgabe der Pessach-Haggadah des Gerschom Kohen, Prag $5287 / 1527$.

Die Pessach-Haggadah, im Dezember 1526 in Prag veröffentlicht und die früheste und einzige vollständig erhaltene illustrierte Haggadah, erschien 400 Jahre nach 
ihrer Erstveröffentlichung bei Josef Altmann. Loewe hatte Altmann bereits im Vorfeld der Gründung der Soncino-Gesellschaft über seine damalige „Schülerin“ Jenny Wilde kennengelernt, ${ }^{38}$ die lange Zeit als Bibliothekarin an der Hochschule für die Wissenschaft des Judentums arbeitete. In den 1920er Jahren musste Altmann sein Geschäft aufgeben, so dass die Pessach-Haggadah der einzige Band der „Monumenta“ blieb, der jemals veröffentlicht wurde.

Die Buchreihe hatte das Ziel, „die älteste hebräische Literatur in photographischen Wiedergaben [...] und zwar, soweit es nur irgend möglich ist, die Erstausgaben“" ${ }^{39}$ zu versammeln. Der Forschung wolle man damit jüdische Quellen zur Verfügung stellen, die frei von „unbeabsichtigten Entstellungen“ und von zensurbedingten Eingriffen in den Jahrhunderten wären. ${ }^{40}$ Loewe hatte sich zu diesem Zeitpunkt offenbar längst mit der Geschichte der Zensur von jüdischer Literatur beschäftigt. In einem mehrseitigen, mit reichlich handschriftlichen Notizen versehenen Manuskript beschreibt er jene in Ansätzen, führt sie bis in die biblische Zeit zurück. ${ }^{41}$ Vergleichbar seiner in einer Vielzahl von Beiträgen entwickelten, dezidiert zionistischen Geschichtsschreibung und Sprachforschung, die auf die Kontinuität jüdischer Geschichte mit gemeinsamer Sprache, dem Hebräischen, abzielend, die Einheit des Judentums konstruierte, ${ }^{42}$ liest er die Geschichte der Zensur jüdischer Bücher als eine „Geschichte seit jeher“. Die jahrhundertelange Verfolgung der Hebräischen Bibel, die Loewe, ganz dem zionistischen Diskurs verhaftet, als „Buch des Volkes“ porträtiert, stellt sich als ,Ur-Katastrophe“ dar. Dieser Topos der Katastrophe ist in einem allgemeineren Sinne bereits Teil des zionistischen Narratives um die Wende vom 19. zum 20. Jahrhundert. Er materialisierte sich etwa als „Verelendungstheorie“433 des Diaspora-Judentums schon in der 1885 von Nathan Birnbaum begründeten Zeitschrift Selbst-Emancipation, deren Chefredaktion Loewe Mitte der 1890er Jahre übernahm. ${ }^{44}$ Vor diesem Hintergrund erstaunt es kaum, dass man durchweg Klassiker der hebräischen Literatur für die

38 Loewe, Heinrich: Sichronot. Kap. Jenny Wilde. CZA, A146/71, S. 2. Jenny Wilde wurde von den Nationalsozialisten offensichtlich Anfang der 1940er Jahre nach Theresienstadt (Terezín) deportiert. Dort wurde sie als wissenschaftliche Bibliothekarin weiterhin eingesetzt, siehe Brief von Jenny Wilde an Heinrich Loewe vom 03.04.1946. CZA, A146/135. Zur „Ghetto-Bibliothek in Theresienstadt“ siehe das gleichnamige Kapitel in Kirchhoff, Häuser des Buches, S. 138-141.

39 Brief von Heinrich Loewe an Victor Avigdor Aptowitzer vom 06.06.1924. Archiv der JNUL, ARC 40, 1209/122.

40 Werbebrief „Erstdrucke der Hebraeischen Literatur“, 1924. BA, offener Bestand.

41 Loewe, Heinrich: Das hebräische Buch unter den Händen der Zensur, o. J. BA, Boxnr. 27.

42 Siehe hierzu Schlöffel, Heinrich Eljaqim Loewe, Kap. 6.2 „Die Sprache(n) der Juden“.

43 Margulies, Heinrich: Kritik des Zionismus, Bd. 2. Wien 1920, S. 21.

44 Siehe hierzu Schlöffel: Heinrich Eljaqim Loewe, Kap. 3.2 „Die Selbst-Emancipation zirkulieren!“. 
Monumenta Hebraica in Aussicht nahm. ${ }^{45}$ Als Antwort auf Bialiks oben zitierte Zeitdiagnose von einer wenigstens teilweise prekären ,Gegenwart des jüdischen Buches‘, zugleich als Geste des ,Rück-Holens“ jener ,alten Literatur‘ in das ,kollektive jüdische Bewusstsein‘ ließe sich diese Entscheidung interpretieren, die sich bereits im Titel der Buchreihe andeutet: Ein zeitloses ,Monument‘, ein Denkmal und Archiv authentischer jüdischer Literaturproduktion intendierten die Herausgeber, dessen Teil auch die später in anderem Kontext von der Gesellschaft publizierten Soncino-Bibel von 1485 war.

Die Pessach-Haggadah fehlte allerdings unter den zuerst geplanten Bänden. Ein Brief Loewes an Alexander Marx, Bibliothekar am Jewish Theological Seminary in New York, bestärkt die Annahme, dass die Herausgabe der Haggadah eher Notlösung als stringent von Anfang an im Kreise des Soncino-Vorstands geplant war. Zumal mit der Herausgabe von „19 Abbildungen aus dem ersten Holzschnittdruck der Passah-Hagada“ durch den Kunsthistoriker, späteren Kustos der Kunstsammlung der jüdischen Gemeinde sowie Leiter des Jüdischen Museums in Berlin und des Tel Aviv Museum of Art, Karl Schwarz, der Kohen-Haggadah im Gegensatz zu anderen projektierten Drucken bereits 1920 zur größeren öffentlichen Sichtbarkeit verholfen war. ${ }^{46}$ Ursprünglich sollte nämlich an einer Reproduktion des Soncino-Talmuds gearbeitet werden, der als erster Band der Reihe erscheinen sollte. Das Unternehmen erwies sich aber Loewe zufolge in einer Zeit „wirtschaftlicher Nöte in Deutschland“ als zu kostspielig. ${ }^{47}$

Gemeinsam mit Benzion Katz machte sich Loewe kurz nach der konstituierenden Sitzung der Soncino-Gesellschaft an die Vorarbeiten für die Reihe und für die Ausgabe der Kohen-Haggadah im Besonderen. Am 6. Juni 1924 versandten Loewe und Katz erste Briefe an jüdische Wissenschaftler aus ganz Europa, die bei der Herstellung des kritischen Kommentars zu den einzelnen Traktaten der Haggadah behilflich sein könnten - in den folgenden Wochen wurden weitere Zirkulare herausgeschickt: Victor Aptowitzer (Wien), Aron Freimann (Frankfurt am Main), Meier Hildesheimer (Berlin) und Max Wiener (Stettin) sagten wegen ihrer zeitlichen Belastung mit anderen Arbeiten, gesundheitlicher Probleme oder des Gefühls, der falsche Ansprechpartner für ein derartiges Projekt zu sein, die Mitarbeit

45 Siehe hierzu Werbebrief „Erstdrucke der Hebraeischen Literatur“, 1924. BA, offener Bestand. 46 Die Prager Hagada von 1526. 19 Abbildungen aus d. ersten Holzschnittdruck d. Passah-Hagada (= Jüdische Bücherei, Bd. 3, hrsg. v. Karl Schwarz). Berlin 1920. In der von der Berliner Zionistischen Vereinigung herausgegebenen Berliner Jüdischen Zeitung äußerte sich Karl Schwarz Anfang 1932 betont kritisch in Bezug auf die Entscheidung des Vorstands der Soncino-Gesellschaft, Marcus Behmer mit der Schriftgestaltung der Hebräischen Bibel zu beauftragen. In der folgenden Zeit entspann sich zwischen Schwarz und Meyer ein Schlagabtausch darüber in Briefen. Siehe hierzu den Beitrag von Philipp Messner in diesem Band.

47 Brief von Heinrich Loewe an Alexander Marx vom 03.09.1925. BA, offener Bestand. 
am kritischen Kommentar der Haggadah ab. ${ }^{48}$ Während Heinrich Brody (Prag) und Josef Wohlgemuth (Berlin) unentschlossen blieben, ${ }^{49}$ waren unter Loewes Adressaten auch eine Reihe von Gelehrten, die gern an der Herstellung des Kommentars mitarbeiten wollten, darunter etwa Michael Guttmann und Israel Rabin aus Breslau, Arthur Marmorstein (London), Hanoch Albeck (Wien), Julius Lewy (Gießen), Hugo Greßmann (Berlin-Schlachtensee), Lajos Blau (Budapest) und Umberto Cassuto (Florenz). ${ }^{50}$ Ein Teil der Gelehrten monierte allerdings, dass ihnen von Loewe und Katz kein konkreter Editionsplan vorgelegt wurde. Nichtsdestotrotz trieben die beiden den Herstellungsprozess der Haggadah in den folgenden Monaten voran. Zunächst mussten Druckvorlagen beschafft werden, was sich als schwieriges Unterfangen herausstellte, da von der Kohen-Haggadah weltweit nur noch drei Exemplare - an der Bibliothek des Jewish Theological Seminary in New York, an der Stadtbibliothek in Frankfurt am Main und am British Museum in London - existierten. Es war offenbar Aron Freimann, Mitherausgeber des Mitteilungsblatts der Soncino-Gesellschaft und Leiter der orientalischen Sammlungen in Frankfurt am Main, zu verdanken, dass die Stadtbibliothek ihr Exemplar nach Berlin transferierte. ${ }^{51}$ Auf Vermittlung von Arthur Marmorstein, der seinerzeit am Jew's College unterrichtete, gelang es ferner, das Exemplar des British Museum zu bekommen. Frühestens im Februar 1925 erreichte das Original aus Frankfurt Berlin. ${ }^{52}$ Von dort aus ging es vermutlich für beide Haggadot weiter nach Leipzig, wo die Firma Brockhaus den Druck besorgte. ${ }^{53}$ Bruno Kirschner, der

48 Siehe hierzu Postkarte von Victor Aptowitzer an Heinrich Loewe vom 24.06.1924. BA, Boxnr. 23; Postkarte von Aron Freimann an Heinrich Loewe vom 10.06.1924. BA, Boxnr. 23; Brief von Meier Hildesheimer an Heinrich Loewe vom 12.06.1924. BA, Boxnr. 24; Brief von Max Wiener an Heinrich Loewe vom 22.06.1924. BA, offener Bestand.

49 Siehe hierzu Brief von Heinrich Brody an Heinrich Loewe vom 27.06.1924. BA, Boxnr. 2; Brief von Josef Wohlgemuth an Heinrich Loewe vom 14.07.1924. BA, Boxnr. 26.

50 Siehe hierzu Brief von Michael Guttmann an Heinrich Loewe vom 20.06.1924. BA, Boxnr. 24; Brief von Israel Rabin an Heinrich Loewe und Benzion Katz vom 25.06.1924. BA, Boxnr. 25; Brief von Arthur Marmorstein an Heinrich Loewe vom 16.07.1924. BA, Boxnr. 25; Brief von Hanoch Albeck an Heinrich Loewe und Benzion Katz vom 27.06.1924. BA, Boxnr. 23; Brief von Julius Lewy an Heinrich Loewe vom 08.07.1924. BA, Boxnr. 24; Brief von Hugo Greßmann an Heinrich Loewe vom 12.07.1924. BA, Boxnr. 24; Brief von Lajos Blau an Heinrich Loewe vom 13.07.1924. BA, Boxnr. 23; Brief von Umberto Cassuto an Heinrich Loewe vom 15.07.1924. BA, Boxnr. 23.

51 Vgl. Postkarte von Aron Freimann an Heinrich Loewe vom 16.01.1925. BA, Boxnr. 23.

52 Ebenda.

53 Man druckte im Quart-Format. Die Gesamtauflage der Faksimile-Ausgabe belief sich auf 300 Exemplare. Das auf Van-Gelder-Bütten gesetzte und in Leinen eingeschlagene Buch, welches einen handgefertigten Einband und ein „handumstochenes Kapital“ (Rundschreiben an die Mitglieder der Soncino-Gesellschaft vom 25.03.1926. JMB, Sammlung Soncino-Gesellschaft, DOK 93/502/72) aufwies, wurde in Umschlag und Futteral an die Mitglieder ausgeliefert. Zwei Ausgaben waren verfügbar. Die erste (und zu 16,50 Mark preiswertere) Ausgabe besaß einen „Pappband 
regelmäßig in der Jüdischen Rundschau über die Soncino-Gesellschaft schrieb, fasste die Qualität des Druck-Erzeugnisses wie folgt zusammen:

Es ist ein Werk von unvergleichlicher Schönheit, das der Verlag von Josef Altmann [...] dem kunstsinnigen Publikum vorlegt. Diese Haggadah ohne Mätzchen, ihr einfach-klares architektonisch gegliedertes Satzbild, die monumental gequaderten Konsonanten mit den breitgezogenen dünnen Vokalzeichen, die Anordnung der Anmerkungen und vor allem die Holzschnitte sind eine wahrhafte Erquickung. ${ }^{54}$

Aber auch Kritik wurde laut. Kirschner und zugleich Alexander Marx, der eine kurze Besprechung der Haggadah im Jewish Quarterly Review veröffentlichte, monierten einige Punkte an der Neuausgabe. Die Anmerkungen, die von den von Loewe und Katz eingeladenen Experten abgefasst wurden, seien teilweise unleserlich. ${ }^{55}$ Zurückzuführen sei dies auf abgenutzte Lettern, die von der Leipziger Druckerei benutzt worden wären. Eine Seitenzählung wäre zum Nachteil für die Leser nicht eingefügt worden. Außerdem hätte man in das Originalarrangement der Haggadah eingegriffen. Insbesondere letzterer Kritikpunkt wiegt vor dem Hintergrund, dass die beiden Herausgeber, wie erwähnt, die in den ,Monumenta' erscheinenden Faksimiles unverfälscht abzudrucken gedachten, besonders schwer. Während im Kohen-Druck von 1526 die erste Seite im Innenteil des Buches frei blieb und der Text auf der Rückseite des ersten Blattes begann, rückte man die erste Seite des Druckes der Soncino-Gesellschaft eine Seite nach vorne und begann mit dem Text auf der Vorderseite des ersten Blattes. „To my taste“, kommentierte Marx, „the effect of the original arrangement is much finer and more artistic." 56

mit Pergament-Verstärkung an den Ecken und Kapitalen mit Kopfschnitt“, die zweite Ausgabe zu 18 Mark war mit Halbpergament bezogen und hatte einen Kopfgoldschnitt (vgl. ebenda).

54 Kirschner, Bruno: Adel des Buches. In: Jüdische Rundschau 72 (11.09.1925), S. 612-613.

55 Vgl. ebenda; Marx, Alexander: Two Illustrated Haggadah. In: The Jewish Quarterly Review 16, 4 (1926), S. 471-474, hier S. 471.

56 Ebenda. 